\title{
ONTOLOGY DEVELOPMENT FOR WHEAT INFORMATION SYSTEM
}

\author{
Sumit Kumar Mishra' ${ }^{1}$, V.K. Singh ${ }^{2}$, Gaurav Kant Shankhdhar ${ }^{3}$ \\ ${ }^{1}$ Pursuing M.Tech, Department of Software Engineering, Babu Banarasi Das University, Lucknow, UP, India, \\ ${ }^{2}$ Assoc. Prof. \& Head - I.T., Babu Banarasi Das National Institute of Technology \& Management, Lucknow. India \\ ${ }^{3}$ Assistant Professor, School of Computer Application, BBDU
}

\begin{abstract}
Ontology Development For Wheat Information System makes use of the semantic web and it used for valid Wheat information retrieve which help for agriculture Insurance policies and other information like time of harvesting, condition of soil is appropriate for wheat farming or not. In this way we can better prepare himself with similar cases of wheat species .the role of web semantics here is that we introduced intelligent matching of wheat information. The search is not only through but also accurate and precise to the maximum level of attainment with the use of ontology designed exclusively for this purpose. the project Ontology development for wheat information helps the machine to take appropriate decision regarding symptoms also.
\end{abstract}

Keywords -RDF, SPARQL, Web Semantic, Wheat Diseases

\section{INTRODUCTION}

In India basically two most important food Wheat and rice but wheat is primary food for India. Wheat normally needs between 110 and 130 days between Sowing and harvesting depending upon climate, seed type, soil conditions etc. In Ontology development for Wheat Information System we develop Wheat ontology and apply this ontology to information retrieval mechanism as a knowledge base for retrieving and managing acquaintance in a field of agriculture.[1]

\subsection{Wheat Classification}

There are 6 wheat classification are given below
a) Hard Red Winter
b) Hard Red Spring
c) Soft Red Winter
d) $\operatorname{Durum}($ Hard $)$
e) Hard White
f) Soft White Wheat[2]

\subsection{Wheat Diseases}

Wheat diseases are classified in 4 types:

- Bacterial

- Fungal

- Viral

- $\quad$ Phytoplasmal [3]

\subsection{Proposed System}

The Proposed System primarily consists of the classes, properties or the predicates in connection to the RDF and the individuals that are the objects instantiated through classes. The Wheat Ontology is built in Protégé 4.3. This ontology provides for the framework of the Wheat ontology System. DotNetRDF which is a RDF API used in Microsoft Visual Studio for implementing Semantic Web Solution is extensively exploited over here. A SPARQL query is submitted to the DotNetRDF API which in conjunction with ASP.NET provides results as queried by the SPARQL interface. So the request and response is handled by the system.

\section{WHEAT CASE ONTOLOGY DESIGN}

Wheat case ontology is based on two combination model that is dependent and independent semantics .Basically ontology consists 4 tuples $\langle\mathrm{C}, \mathrm{I}, \mathrm{R}, \mathrm{A}\rangle$ to design basic ontology we define all tuples.[4,5]

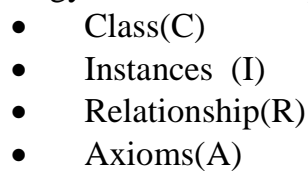

\subsection{Classes and Properties of Wheat Ontology}

A class provides an abstraction mechanism for grouping resources with same type characteristics [6], whilst a property is often used to identify the non hierarchical relationships between domain and range (denoted as $R$ (domain, range)).OWL defines two types of properties: data property and object property. Data property is an alias of attribute while object property is a binary relationship between two classes.

\subsection{Classes of Wheat Ontology}

Wheat ontology shown relationship between super class and subclass and Things represent the main wheat information system. In given diagram Wheat crop is super class this super class linked with given some sub class that is:

Table1. Wheat Sub Class

\begin{tabular}{|l|}
\hline Wheat_Classification \\
\hline Scientific_classification \\
\hline Harvesting \\
\hline Production_technology \\
\hline
\end{tabular}




\begin{tabular}{|l|}
\hline Species \\
\hline Soil \\
\hline Climate \\
\hline Marketing \\
\hline Wheat_diseases \\
\hline Economics \\
\hline
\end{tabular}

\subsection{Properties}

Properties are instances of the class rdf:Property. In the RDF graph, the property represents the predicate and describes a relation between subject resources and object resources. [7,8]

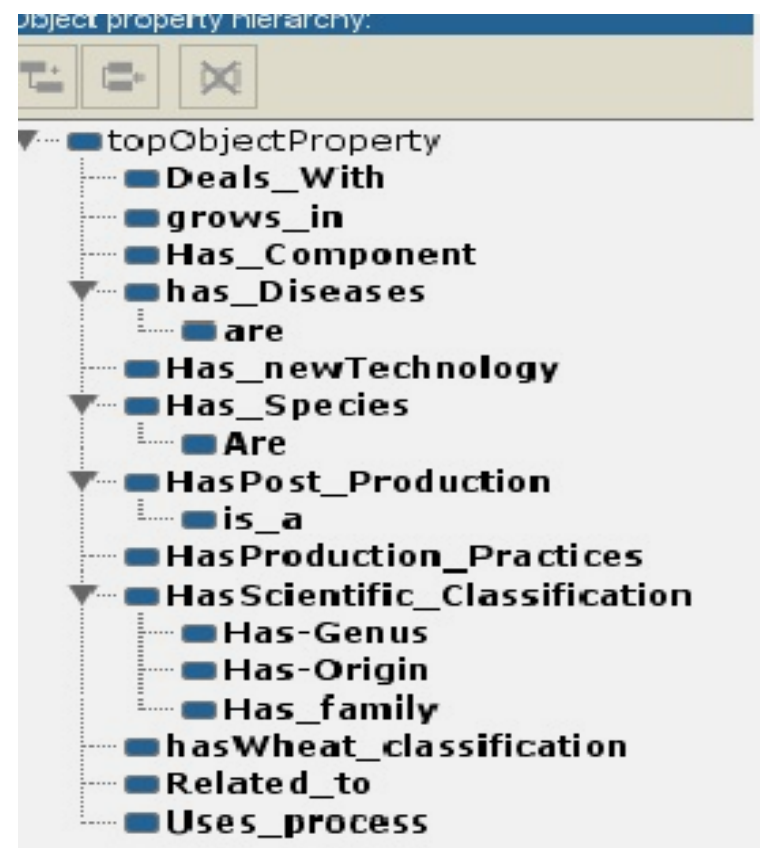

Fig.1 Object Properties of Wheat Ontology

\section{CLASS HIERARCHY OF WHEAT ONTOLOGY SYSTEM}

In this ontology we provide basic hierarchy between super class and sub class.[9,10] theses classes linked with other subclass, In RDF graph theses class describes basic ontology features and connection to other subclass or sibling class. In Wheat ontology one is super class i.e. parent class of all classes. This class known as Thing Class.

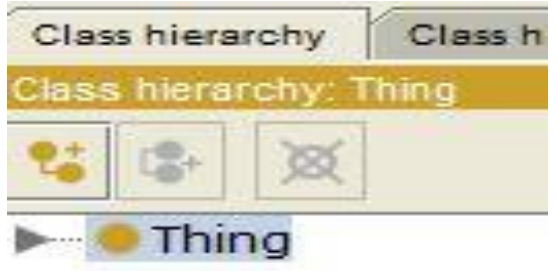

\section{WHEAT ONTOLOGY INDIVIDUALS}

the individuals that are the objects instantiated through classes. In Wheat Ontology Individuals Role is most important with the help of these Individuals we retrieve information in any wheat species Wheat Individuals we use basic names of wheat

Ex. H.P.1731,NARENDRA Wheat etc.

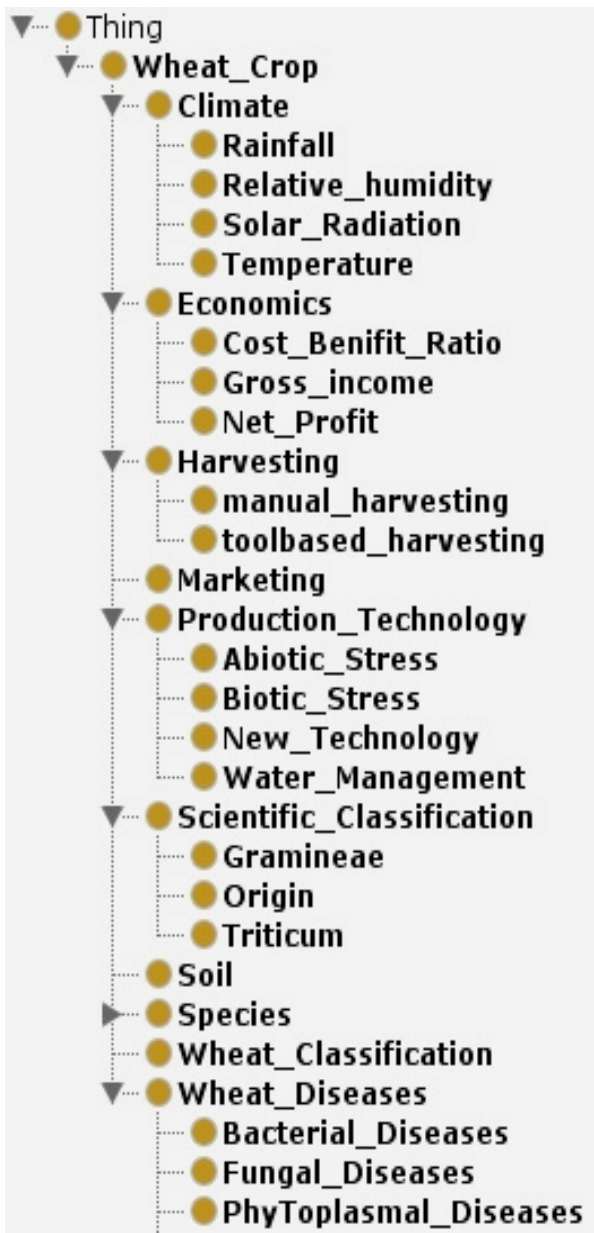

Fig .3 Class Hierarchy Of Wheat Ontology

Fig 2 Thing class 


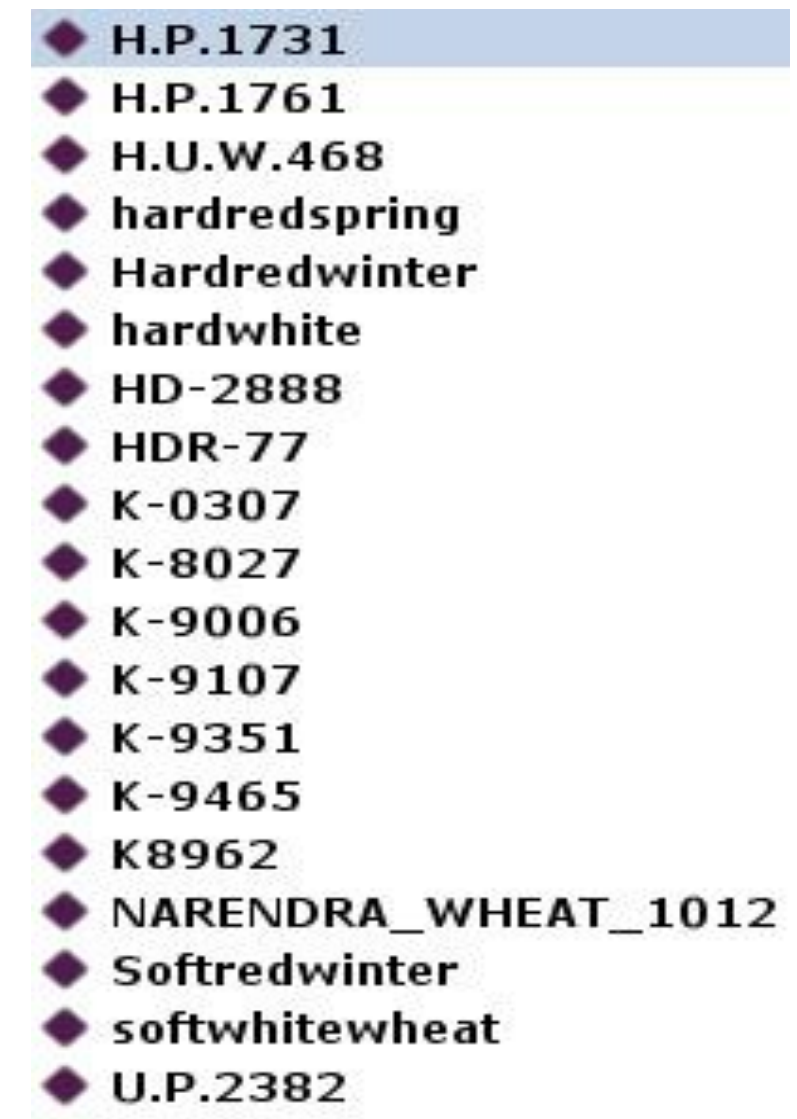

Fig.4 Wheat Individuals

\section{SPARQL}

SPARQL is used to query the RDF file. It is quite similar to SQL which is used to query RDBMSs. RDF provides great ways to model and store data, and the Linked Data infrastructure offers tons of data to play with. As long as $\mathrm{RDF}$ has been around, there have been programming libraries that let you load triples into the data structures of popular programming languages so that you could build applications around that data. As the relational database and XML worlds have shown, though, a straightforward query language that requires no compiling of code to execute makes it much easier for people (including part-time developers dabbling in the technology) to quickly assemble applications.[11]

\subsection{SPARQL Query for related to Wheat Ontology}

PREFIX rdf: <http://www.w3.org/1999/02/22-rdf-syntaxns\#>

PREFIX owl: <http://www.w3.org/2002/07/owl\#>

PREFIX xsd: <http://www.w3.org/2001/XMLSchema\#>

PREFIX rdfs: <http://www.w3.org/2000/01/rdf-schema\#> SELECT $*$

where \{ ?element

<http://www.semanticweb.org/rs/ontologies/2014/9/Wheat case-25\#hasSpeciesNum>?SPC_NUM.

?element

<http://www.semanticweb.org/rs/ontologies/2014/9/WheatC ase-25\#hasSectionID> ?Related_Sec_ID.

\{ SELECT ?Related_Sec_ID

where

\{

?element

<http://www.semanticweb.org/rs/ontologies/2014/9/WheatC ase-25\#hasRelatedSEC_ID $>$ ?Related_Sec_ID.

?element

<http://www.semanticweb.org/rs/ontologies/2014/9/WheatC ase-25\#hasSpeciesNum>

$<$ http://www.semanticweb.org/rs/ontologies/2014/9/WheatC ase-25\#3>.

\}
\}

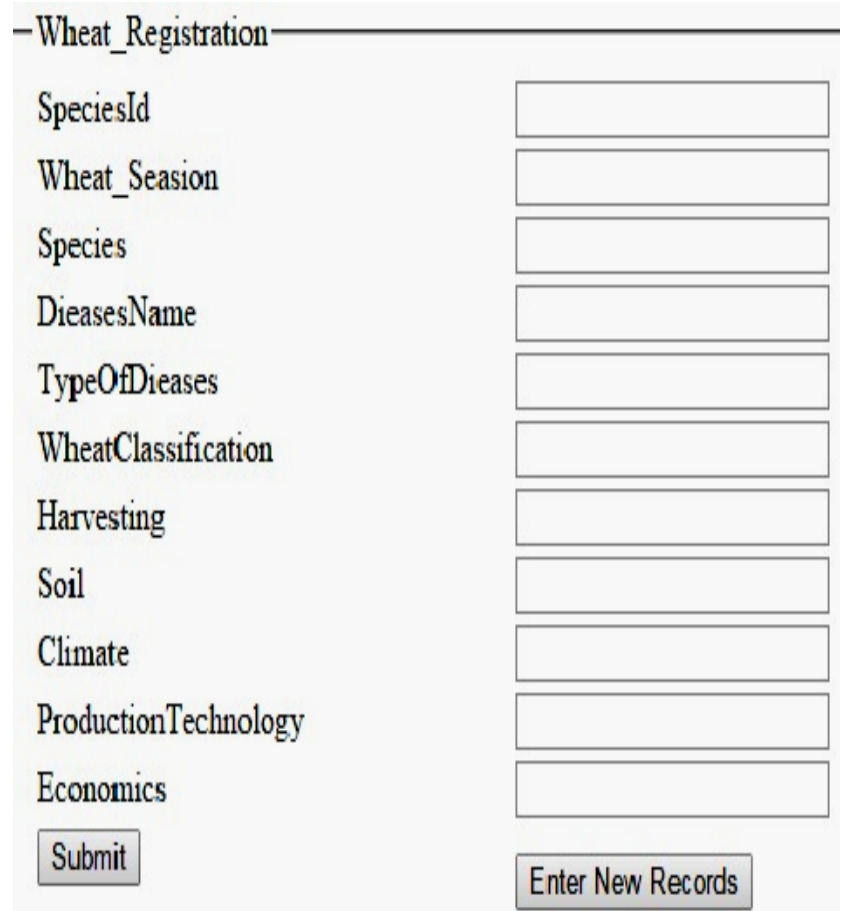

Fig 5 Wheat Registration Information Page

\section{RDF FILE CODE FOR WHEAT INFORMATION SYSTEM}

$<$ ?xml version="1.0" encoding="utf-8"?>

$<$ !DOCTYPE rdf:RDF [

$<$ !ENTITY rdf 'http://www.w3.org/1999/02/22-rdf-syntaxns\#'>

$<$ !ENTITY rdfs 'http://www.w3.org/2000/01/rdf-schema\#'> $<$ !ENTITY xsd 'http://www.w3.org/2001/XMLSchema\#'> ]>

<rdf:RDF Xmlns:rdfs="http://www.w3.org/2000/01/rdfschema\#"

xmlns:xsd="http://www.w3.org/2001/XMLSchema\#" 
xmlns:rdf="http://www.w3.org/1999/02/22-rdf-syntax-

ns\#">

$<$ rdf:Description

rdf:about="http://www.semanticweb.org/dell/ontologies/201

5/0/untitled_ontology-6\#SpeciesIdafa14055-6a5a-45f2-

b233-7c95d5f3ca74">

$<$ ns0:Classification

xmlns:ns0="http://www.semanticweb.org/dell/ontologies/20

15/0/untitled_ontology-6\#" >fungal </ns0:Classification $>$

$<$ ns 1:Climate

xmlns:ns1="http://www.semanticweb.org/dell/ontologies/20

15/0/untitled_ontology-6\#">lowsoil</ns1:Climate>

$<$ ns2:DieasesName

xmlns:ns2="http://www.semanticweb.org/dell/ontologies/20

15/0/untitled_ontology-6\#">redWheat</ns2:DieasesName>

$<$ ns3:Economics

xmlns:ns3="http://www.semanticweb.org/dell/ontologies/20

15/0/untitled_ontology-6\#">manual</ns3:Economics $>$

$<$ ns4:Harvesting

xmlns:ns4="http://www.semanticweb.org/dell/ontologies/20

15/0/untitled_ontology-6\#">orbit</ns4:Harvesting>

$<$ ns5:ProductionTechnology

xmlns:ns5="http://www.semanticweb.org/dell/ontologies/20

15/0/untitled_ontology-

6\#">lesstype</ns5:ProductionTechnology $>$

$<$ ns6:Soil

xmlns:ns6="http://www.semanticweb.org/dell/ontologies/20

15/0/untitled_ontology-6\#">manual</ns6:Soil>

$<$ ns7:SpeciesId

xmlns:ns7="http://www.semanticweb.org/dell/ontologies/20

15/0/untitled_ontology-6\#">afa14055-6a5a-45f2-b233-

7c95d5f3ca74</ns7:SpeciesId $>$

$<$ ns8:TypeOfDieases

xmlns:ns8="http://www.semanticweb.org/dell/ontologies/20

15/0/untitled_ontology-

6\#">beactirial</ns8:TypeOfDieases $>$

$<$ ns9:WheatSeasion

xmlns:ns9="http://www.semanticweb.org/dell/ontologies/20

15/0/untitled_ontology-6\#">1</ns9:WheatSeasion>

$<$ ns10:species

xmlns:ns10="http://www.semanticweb.org/dell/ontologies/2

015/0/untitled_ontology-6\#" >winteroctum </ns10:species $>$

$</$ rdf:Description $>$

$</$ rdf:RDF $>$

\section{WORKING OF SOFTWARE}

The interface looks like a search engine in which comma separated values are entered. The semantic, meaning is extracted from the search terms and a semantic search is performed where result is prepared on the basis of the logical meaning of the search terms. Like if somebody searches for "NARENDRA WHEAT", also gets results consisting of searches like "SOIL TYPE", "HARVESTING Information" and more information of wheat.

\section{CONCLUSION AND FUTURE SCOPE}

The future scope of our work is to apply the potential of Knowledge Representation[12,13,14] along with reasoning in the Web context. The use of semantic web in crop Wheat
Information System helps the machine to take the appropriate decision regarding symptoms and cure. In future scope we develop an prototype Wheat ontology that integrate agriculture domain and semantic web. With the help of this ontology farmer retrieve information and check proper condition for harvesting and climate.

\section{REFERENCES}

[1]. Sumit Kumar mishra, Dr. V.K. Singh Anurag Tiwari“'Ontology development for agriculture research a case study of wheat " journal of basic and applied engineering research.

[2]. Six Basic Classes of Wheat Minnesota Association of Wheat Growers.

[3]. Chalkley , D,(2010), " Invasive Fungi: Alternaria leaf blight of Wheat -Alternaria triticina". Systematic mycology and Microbiology laboratory, Agricultural Research Service. Unfitted States Department Of Agriculture Archived From the original on 29 October 2014.

[4]. Natalya F. Noy and Deborah L. McGuinness,

Ontology Development 101: A Guide for Creating Your First Ontology.

[5]. Toby Segaran, Colin Evans, and Jamie Taylor, -Programming the Semantic Web.

[6]. Gómez-Pérez, A., Fernández-López, M., Corcho, O. Ontological Engineering, with examples from the areas of Knowledge Management, e-Commerce and the Semantic Web. Springer, London, Berlin (2003)

[7]. Opijnen, Marc van, The European Legal Semantic Web: Completed Building Blocks and Future Work (November 22, 2012). European Legal Access Conference, November 2012.

[8]. Amit Sheth,Cartic Ramakrishnan, and Christopher Thomas, 'Semantics for The Semantic Web: the Implicit, the Formal and the Powerful',International Journal on Semantic Web \& Information Systems, 1 (no. 1), 2005, pp. 1-18.

[9]. 3. Brachman , R.J., McGuinness, D.L., Patel-Schneider, P.F., Resnick, L.A. and Borgida, A. (1991). Living with CLASSIC: When and how to use KL-ONE-like language. Principles of Semantic Networks. J. F. Sowa, editor, Morgan Kaufmann: 401-456

[10]. Legal Semantic Web- A Recommendation System Gaurav Kant, V.K.Singh, M. Darbari, D. Yagyasen ${ }^{4}, P . K$. Shukla International journal ICANI

[11]. Bob DuCharme, Learning SPARQL, O’REILLY,2011. [12]. Gómez-Pérez, A., Fernández-López, M., Corcho, O. Ontological Engineering, with examples from the areas of Knowledge Management, e-Commerce and the Semantic Web. Springer, London, Berlin (2003)

[13]. D Yagyasen, M Darbari, P K Shukla, V K Singh,(2013). "Diversity and Convergence Issues in Evolutionary Multi- objective Optimization: Application to Agriculture Science", IERI Procedia, Elsevier.

[14]. D Yagyasen, M Darbari, H Ahmed, (2013). "Transforming Non-Living to Living: A Case on Changing Business Environment”, 2013, IERI Procedia, Elsevier. 


\section{BIOGRAPHIES}

Sumit Kumar Mishra received his B.Tech degree from G.B.T.U. in 2013. Currently he is pursuing M.Tech in Software engineering from Babu Banarasi Das University, Lucknow Uttar Pradesh, India.

Dr. V.K. Singh Assoc. Prof. \& Head - I.T., Babu Banarasi Das National Institute of Technology \& Management,Lucknow. India.

Gaurav Kant Shankhdhar Assistant Professor, School of Computer Application, BBDU. 